\title{
OPTIMAL MULTIUSER CDMA DECODER USING GROUPED COORDINATE ASCENT AND THE DWT
}

\author{
Man Sharfer \\ Alfred O. Hero \\ Dept. of Electrical Engineering and Computer Science \\ The University of Michigan, Ann Arbor, MI 48109
}

\begin{abstract}
It is well known that direct implementation of the optimal multiuser receiver for an asynchronous Code Division Multiple Access (CDMA) communication system has an exponential complexity in the number of users. In this paper we present an iterative implementation of an optimal multiuser receiver which has at most cubic complexity per iteration in the number of users. The receiver performs joint Maximum Likelihood (ML) estimation of the amplitudes, phases, time delays, and symbol sequences of all the users. Similarly to the single user receiver we presented in [1], the multiuser receiver is based on a signal decomposition with respect to a compactly supported orthonormal wavelet basis. The algorithm uses grouped coordinate ascent updates to increase the likelihood function in each iteration. Simulation results of a two user system are presented showing that the receiver is near-far resistant, and attains the single user bit error probability bound.
\end{abstract}

\section{INTRODUCTION}

In an asynchronous CDMA system several users transmit simultaneously over the same frequency band, each modulating a pre-assigned wideband Pseudo Noise (PN) sequence. Due to the unsynchronized nature of the transmissions it is not possible to guarantee strict orthogonality between the users for all relative delays and modulating symbol sequences. In the absence of orthogonality the performance of the of the conventional receiver (matched filter) severely deteriorates, especially when some of the interfering users have energies that are much higher than the energy of a particular user. This problem, called the near-far problem, motivated the recent work on multiuser receivers that are robust to this effect, provide close to optimal performance, and have low complexity. The optimal multiuser receiver, in the sense of jointly maximizing the likelihood of the transmitted symbol sequences of all the users, was studied in [3]. The optimal receiver has an exponential complexity in the number of users, requires the knowledge of user energies and time delays, and has a large decoding delay, so it is generally impractical. Suboptimal receivers with a linear complexity in the number of users, such as the decorrelating receiver [4], have been investigated. Receivers of this type are near-far resistant, but they enhance the noise.

In this paper we present an iterative solution to the ML parameter estimation problem, where the unknown param- eter consists of the complex gains (amplitude and phase), time delays, and symbol sequences of each of the users. This approach is the most general since estimation of the gain and delay parameters is essential for implementing a phase coherent and time synchronized receiver. Maximization of the likelihood function for the Additive White Gaussian Noise (AWGN) channel is analytically intractable. To solve this problem we use an iterative Grouped Coordinate Ascent (GCA) algorithm, in which a subset of the parameter is updated in each iteration. We derive an efficient digital algorithm that can be executed in real time, has a fixed complexity, and only requires a "single pass" over the received signal. The GCA algorithm is related to a class of EM algorithms known as Space Alternating Generalized EM (SAGE) algorithms [2], and like SAGE its asymptotic convergence rate is significantly better than standard EM.

The implementation of the algorithm is facilitated by expanding the signal on a time-frequency localized basis. This expansion allows us to develop a simple recursion from one cycle of updates to the next one, and to effectively decouple the parameter updates. The choice of a compactly supported orthonormal wavelet basis [5] is motivated by its excellent time-frequency localization, multi resolution, and the existence of efficient algorithms for computing the Discrete Wavelet Transform (DWT) via discrete time filter banks. The algorithm has at most cubic complexity (typically quadratic complexity) per iteration in the number of users, and involves tractable updates of all the parameters; time delays are updated via polynomial rooting, data symbols are updated by a simple discrete search or via unconstrained maximization of a quadratic form, and complex gains are updated analytically.

This paper is organized as follows. In section 2 we define the multiuser system model and introduce the GCA algorithm. In Section 3 we describe the wavelet expansion and outline the parameter update strategy. The efficient implementation of the algorithm is given in Section 4 . We conclude with simulation results of a two user system.

\section{SYSTEM MODEL AND THE GCA ALGORITHM}

We consider the following complex baseband model of an $L$ user CDMA system:

$$
y(t)=\sum_{l=0}^{L-1} s_{l}(t)+u(t), \quad-\infty<t<\infty
$$


where $s_{l}(t)$ is an attenuated and delayed version of the data modulated signal transmitted by the $l$-th user,

$$
s_{l}(t)=a_{l} \sum_{n=0}^{N-1} b_{n, l} p_{l}\left(t-n T_{p}-d_{l}\right) .
$$

The unknown parameters associated with the $l$-th user are the complex gain $a_{l} \in\left\{R e^{i \varphi}: R>0,0 \leq \varphi<\pi\right\}$, the data symbols $b_{n, l} \in\{ \pm 1\}$ for $n=0, \ldots, N-1$, and the time delay $d_{l} \in\left[-T_{d} / 2, T_{d} / 2\right]$ for some positive $T_{d}$. The signaling waveforms $p_{l}(t)$ are known $P N$ sequences compactly supported on $\left[-T_{s} / 2, T_{s} / 2\right]$ where $T_{s} \geq T_{p}$. Usually it is assumed that $T_{s} \approx T_{p}$, however this assumption is not explicitly used here. The data symbol model corresponds to a BPSK modulation format, but with minor modifications the algorithm can also be applied to M-ary PSK, PAM, or QAM. The restriction on the phase of the complex gains $a_{l}$ is necessary in order to avoid the phase ambiguity in the signal model (2). Without loss of generality we assume that all the complex gains are restricted to the upper half of the complex plane. The noise $u(t)$ is a complex, zero mean, white Gaussian process with power spectral density $N_{0} / 2$. The goal is to find the ML estimate of the parameter vector $\theta$ given the observation $y=\{y(t):-\infty<t<\infty\}$, where $\boldsymbol{\theta}=\left[\boldsymbol{\theta}_{0}, \ldots, \boldsymbol{\theta}_{L-1}\right]^{T}$ and $\boldsymbol{\theta}_{l}=\left[a_{l}, d_{l}, b_{n, l}: n=0, \ldots, N-1\right]^{T}$.

The GCA algorithm is a simple iterative algorithm for finding the Penalized Maximum Likelihood (PML) estimate of $\theta$ given the observation $Y=y$ :

$$
\hat{\theta}=\arg \max _{\theta \in \Theta} \Phi(\theta)
$$

where

$$
\phi(\boldsymbol{\theta})=\log p(y ; \boldsymbol{\theta})-P(\boldsymbol{\theta}),
$$

and $P(\theta)$ is a suitably defined penalty function. The elements of $\theta$ are indexed by the set of integers $I_{K}=$ $\{0, \ldots, K-1\}$. At each iteration an index set $S \subset I_{K}$ is chosen and the objective (4) is maximized with respect to $\boldsymbol{\theta}_{S}$ which denotes the elements of $\boldsymbol{\theta}$ indexed by $S$, while keeping $\theta_{\bar{S}}$ fixed where $\bar{S}$ is the complement of $S$.

\section{EXPANSION ON AN ORTHONORMAL WAVELET BASIS}

The effectiveness of the recursive algorithm is enhanced by choosing a wavelet basis representation, because it requires a small number of basis coefficients, and it yields an algorithm with rapid convergence. It is superior to other choices, e.g. a windowed Fourier basis which has poor frequency localization. Let $\psi_{j k}(t)$ be an orthonormal wavelet basis, where $\psi(t)$ is the basic wavelet and $\psi_{j k}(t)=\psi\left(2^{-j} t-k\right)$. The received signal $y(t)$ is expanded on this basis, yielding a set of wavelet coefficients:

$$
y_{j k}=\int y(t) \psi_{j k}(t) d t, \quad j, k \in \mathbb{Z} .
$$

Using the multiuser system model $y_{j k}$ are given by:

$$
y_{j k}=\sum_{l=0}^{L-1} a_{l} \sum_{n=0}^{N-1} b_{n, l} w_{j k}(n, l ; d)+u_{j k}, \quad j, k \in \mathbb{Z},
$$

where

$$
w_{j k}(n, l ; d)=\int p_{l}\left(t-n T_{p}-d\right) \psi_{j k}(t) d t
$$

are the wavelet coefficients associated with the $l$-th user's signaling waveform, and $u_{j k}$ are the iid Gaussian noise random variables. This can also be written in terms of the Fourier decomposition of $w_{j k}(n, l ; d)$ as:

$$
y_{j k}=\sum_{i=0}^{L-1} a_{l} \sum_{n=0}^{N-1} b_{n, l} c_{j k}^{T}(n, l) z_{l}+u_{j k}, \quad j, k \in \mathbb{Z}
$$

where $\boldsymbol{c}_{j k}(n, l)=\left[\left\{c_{j k, m}(n, l): m \in \mathbb{Z}\right\}\right]^{T}, \boldsymbol{z}_{l}=\left[\left\{z_{l}^{m}: m \in\right.\right.$ $\mathbb{Z}\}]^{T}, c_{j k, m}(n, l)$ are the Fourier coefficients of $w_{j k}(n, l ; d)$ on the interval $\left[-T_{0} / 2, T_{0} / 2\right]$ :

$$
c_{j k, m}(n, l)=\frac{1}{T_{0}} \int_{-T_{0} / 2}^{T_{0} / 2} w_{j k}(n, l ; \delta) e^{-i m \omega_{0} \delta} d \delta,
$$

and $z_{l}=e^{i \omega_{0} d_{l}}, \omega_{0}=2 \pi / T_{0}$. In a similar fashion to the single user algorithm we define the convolution vector $d_{j k}\left(n_{1}, n_{2}, l\right)=\left[\left\{d_{j k, m}\left(n_{1}, n_{2}, l\right): m \in \mathbb{Z}\right\}\right]^{T}$ for each user, where

$$
d_{j k, m}\left(n_{1}, n_{2}, l\right)=\sum_{p \in \mathbb{Z}} c_{j k, p}\left(n_{1}, l\right) c_{j k, p-m}^{*}\left(n_{2}, l\right) .
$$

Next we define an increasing sequence of wavelet index sets $W^{(s)}, s \in \mathbb{N}$, each is a subset of $\mathbb{Z}^{2}$. The recursive algorithm is organized in cycles indexed by $s$, and iterations indexed by $i$. In cycle $s$ the algorithm uses only those wavelet coefficients whose indices are in $W^{(s)}$, and performs several iterations (updates) on subsets of $\theta$ such that the likelihood is increased. Finally we define the following variables:

$$
\begin{aligned}
\mu^{(s)}(n, l) & =\sum_{j k \in W^{(s)}} y_{j k} c_{j k}(n, l), \\
\nu^{(s)}\left(n_{1}, n_{2}, l\right) & =\sum_{j k \in W^{(s)}} d_{j k}\left(n_{1}, n_{2}, l\right), \\
\sigma^{(s)}\left(n_{1}, n_{2}, l_{1}, l_{2}\right) & =\sum_{j k \in W^{(s)}} c_{j k}\left(n_{1}, l_{1}\right) c_{j k}^{H}\left(n_{2}, l_{2}\right) .
\end{aligned}
$$

The multiuser GCA algorithm is presented in the next section.

\section{MULTIUSER GCA ALGORITHM}

An efficient implementation of the multiuser GCA algorithm is given below. We assume that the algorithm is in the $i+1$-st iteration of the $s+1$-st cycle, so the objectives for the wavelet index set $W^{(s+1)}$ are computed. The state of the parameter estimates at the end of the $i$-th iteration is denoted by the superscript (i), e.g. $z_{l}^{(i)}, b_{n, l}^{(i)}$, and $a_{l}^{(i)}$ for the $l$-th user time delay (represented by a complex variable), data symbols, and gain parameter estimates respectively. We will use the notation $z=\left[\left\{z^{m}: m \in \mathbb{Z}\right\}\right]^{T}$ for a column vector whose components are integer powers of $z$. 
The algorithm is a "sliding window" type algorithm. We define the current symbol index set $T^{(s)}$ such that only symbols whose index $n$ is in $T^{(s)}$ are updated in cycle $s$. Similarly, a set of symbol indices $U^{(s)}$ is defined corresponding to data symbols whose estimates will not be updated after cycle $s+1$. The set $V^{(s)}$ is a two dimensional set of pairs of symbol indices, such that both symbol estimates will not be updated after cycle $s+1$. The size of each of these sets is fixed, so the algorithm has a fixed complexity per iteration.

\section{Multiuser GCA Algorithm}

1. Initialize $\theta^{(0)}$, initialize the variables $\mu, \nu, \sigma, \alpha, \beta, \rho$, $\varepsilon$, and $\tau$ to zero, and set $s=0, i=0$.

2. Choose a wavelet index set $W^{(s+1)}$.

3. For $l, l_{1}, l_{2} \in I_{L}$ update the variables $\mu^{(s)}, \nu^{(s)}$ and $\sigma^{(s)}$ by accumulating on the difference set $D^{(s+1)}=$ $W^{(s+1)}-W^{(s)}$. Only variables whose symbol indices $n$ are in the current symbol index set $T^{(s+1)}$ are updated.

4. For $l, l_{1}, l_{2} \in I_{L}$ update the state variables $\alpha^{(s)}, \beta^{(s)}$, $\rho^{(s)}, \epsilon^{(s)}$, and $\tau^{(s)}$ as follows:

$$
\begin{aligned}
\alpha^{(s+1)}(l) & =\alpha^{(s)}(l) \\
& +\sum_{\left(n_{1}, n_{2}\right) \in V^{(s)}} b_{n_{1}, l}^{(i)} b_{n_{2}, l}^{(i) *} \nu^{(s)}\left(n_{1}, n_{2}, l\right), \\
\beta^{(s+1)}(l) & =\beta^{(s)}(l)+\sum_{n \in U^{(s)}} b_{n, l}^{(i) *} \mu^{(s)}(n, l), \\
+\quad \rho_{\left(n_{1}, n_{2}\right) \in V^{(s)}}^{(s+1)}\left(l_{1}, l_{2}\right)=\rho^{(s)}\left(l_{1}, l_{2}\right) & b_{n_{1}, l_{1}}^{(i)} b_{n_{2}, l_{2}}^{(i) *} \sigma^{(s)}\left(n_{1}, n_{2}, l_{1}, l_{2}\right), \\
\epsilon^{(s+1)}(n, l) & =\epsilon^{(s)}(n, l) \\
& +\sum_{n_{2} \in U^{(s)}} b_{n_{2}, l}^{(i) *} \nu^{(s)}\left(n, n_{2}, l\right), \\
\tau^{(s+1)}\left(n, l_{1}, l_{2}\right) & =\tau^{(s)}\left(n, l_{1}, l_{2}\right) \\
+ & \sum_{n_{2} \in U^{(s)}} b_{n_{2}, l_{2}}^{(i) *} \sigma^{(s)}\left(n, n_{2}, l_{1}, l_{2}\right) .
\end{aligned}
$$

5. Select a parameter index set $S$ and do one of the following:

- If $\boldsymbol{\theta}_{S}=z_{l}$ for some $l \in I_{L}$ then:

Compute the following temporary variables:

$$
\begin{aligned}
\gamma^{(i+1)}(l) & =\left|a_{l}^{(i)}\right|^{2} \alpha^{(s+1)}(l), \\
\delta^{(i+1)}(l) & =a_{l}^{(i) *}\left(\beta^{(s+1)}(l)\right. \\
& \left.-\sum_{l_{1} \neq l} a_{l_{1}}^{(i)} z_{l_{1}}^{(i) T} \rho^{(s+1)}\left(l_{1}, l\right)\right) .
\end{aligned}
$$

Set up the penalized objective for $z=e^{i \omega_{0} d_{l}}$ of the lth user:

$$
\begin{aligned}
\phi_{l}^{(i+1)}(z)= & \frac{2}{N_{0}} \sum_{m \in \mathbb{Z}}\left(\delta_{-m}^{(i+1)}(l)+\delta_{m}^{(i+1) *}(l)\right. \\
& \left.-\gamma_{m}^{(i+1)}(l)\right) z^{m}+\log \Pi(z),
\end{aligned}
$$

where $\Pi(z)$ is a positive penalty function on the unit circle.

Maximize the penalized objective using polynomial rooting:

$$
z_{l}^{(i+1)}=\underset{z=e^{i \omega}}{\operatorname{argmax}}\left\{\phi_{l}^{(i+1)}(z)\right\} .
$$

- If $\theta_{S}=\left\{b_{n, l}: n \in P, l \in Q\right\}$, where $P \subset T^{(s+1)}$ and $Q \subset I_{L}$, then:

Compute the set of $|P| \cdot|Q|$ variables $\varepsilon^{(i+1)}(n, l)$ (see [6]). Compute the set of $(|P| \cdot|Q|)^{2}$ variables:

$$
\begin{aligned}
\chi_{n_{1}, l_{1}, n_{2}, l_{2}}^{(i+1)}= & \left(a_{l_{1}}^{(i)} z_{l_{1}}^{(i) T}\right. \\
& \left.\sigma^{(s+1)}\left(n_{1}, n_{2}, l_{1}, l_{2}\right) z_{l_{2}}^{(i) *} a_{l_{2}}^{(i) *}\right)^{*}
\end{aligned}
$$

Arrange the $\varepsilon$ and $\chi$ variables above in a $|P| \cdot|Q| \times 1$ vector $\varepsilon^{(i+1)}$ and a $|P| \cdot|Q| \times|P| \cdot|Q|$ matrix $\chi^{(i+1)}$ respectively. The objective for $b=\left\{b_{n, l}: n \in\right.$ $P, l \in Q\}$ arranged as a column vector is given by:

$$
\begin{gathered}
\phi^{(i+1)}(b)=\frac{2}{N_{0}}\left[b^{T} \varepsilon^{(i+1)}+b^{H} \varepsilon^{(i+1) *}\right. \\
\left.-b^{H} \chi^{(i+1)} b\right] .
\end{gathered}
$$

Maximize the objective either by a discrete search or via the following suboptimal solution:

- Let $\hat{b}=\operatorname{sign}\left(\left(\chi^{(i+1)}\right)^{-1} \varepsilon^{(i+1) *}\right)$. If $\phi^{(i+1)}(\hat{b}) \geq$ $\phi^{(i+1)}\left(b^{(i)}\right)$ set $b^{(i+1)}=\hat{b}$ else set $b^{(i+1)}=b^{(i)}$.

- If $\boldsymbol{\theta}_{S}=\left[a_{l}, b_{n, l}: l \in I_{L}, n \in I_{N}\right]^{T}$ then:

Compute the elements of the $L \times 1$ vector $\zeta^{(i+1)}$ and the $L \times L$ matrix $\eta^{(i+1)}$ :

$$
\begin{aligned}
& \zeta_{l}^{(i+1)}=z_{l}^{(i) H} \beta^{(s+1)}(l), \\
& \eta_{l_{2}, l_{1}}^{(i+1)}=z_{l_{1}}^{(i) T} \rho^{(s+1)}\left(l_{1}, l_{2}\right) z_{l_{2}}^{(i) *} .
\end{aligned}
$$

The objective for $a=\left[a_{0}, \ldots, a_{L-1}\right]^{T}$ is given by:

$$
\phi^{(i+1)}(a)=a^{H} \zeta^{(i+1)}+\zeta^{(i+1) H} a-a^{H} \eta^{(i+1)} a .
$$

Maximize the objective (19). Let,

$$
\hat{a}=\left(\eta^{(i+1)}\right)^{-1} \zeta^{(i+1)}
$$

For each $l \in I_{L}$, if $0 \leq \arg \left(\hat{a}_{l}\right)<\pi$ set $a_{l}^{(i+1)}=\hat{a}_{l}$ and $b_{n, l}^{(i+1)}=b_{n, l}^{(i)}$ for all $n \in I_{N}$, otherwise set $a_{l}^{(i+1)}=-\hat{a}_{l}, b_{n, l}^{(i+1)}=-b_{n, l}^{(i)}$ for all $n \in I_{N}$, and change the sign of $\beta^{(s+1)}(l),\left\{\rho^{(s+1)}\left(l, l_{2}\right): l_{2} \in\right.$ $\left.I_{L}\right\},\left\{\rho^{(s+1)}\left(l_{1}, l\right): l_{1} \in I_{L}\right\},\left\{\epsilon^{(s+1)}(n, l): n \in\right.$ $\left.T^{(s+1)}\right\}$, and $\left\{\tau^{(s+1)}\left(n, l_{1}, l\right): n \in T^{(s+1)}, l_{1} \in I_{L}\right\}$.

6. Set $\theta_{S}^{(i+1)}=\theta_{S}^{(i)}$.

7. Set $i=i+1$. If cycle is complete proceed to next step, otherwise go back to step 5 .

8. Set $s=s+1$ and go back to step 2 .

The symbol and gain updates require matrix inversions, which have a cubic complexity in $L$ if joint estimation of all the users is done. Typically, smaller groups of users will suffice, so the complexity becomes close to quadratic in $L$. 


\section{SIMULATIONS}

Bit error simulations of the multiuser algorithm were done for a two user system. Each user was assigned a 7 chip Gold code. The data modulated multiuser signal was then passed through a low pass filter with bandwidth $1 / T_{c}$ where $T_{\mathrm{c}}$ is the chip interval.

The simulations were performed in blocks of approximately 250 bits each, where in each block the gains, time delays, data bits and noise were chosen randomly and independently. The data bits $\left\{b_{n, l}\right\}$ were selected as either +1 or -1 , the time delays $d_{l}$ were uniformly distributed in $\left[-T_{p} / 2, T_{p} / 2\right]$, and the gains $a_{l}$ were chosen with a fixed amplitude and random phase uniformly distributed in $[-\pi, \pi]$. The algorithm has to be properly initialized in order to avoid convergence to a local maximum. Typically, the gain and time delay estimates converged within the first 30 bits of each transmission, so they were assumed to be known for the purpose of bit error simulations. Note that the restriction on the phase is no longer necessary for known gains, because the symbols can now be unambiguously estimated.

The performance of the GCA algorithm depends on the parameter update strategy. Updating a single coordinate in each iteration, leads to a rapid convergence but increases the likelihood of the algorithm being trapped in a local maximum. Generally, the convergence rate is inversely related to the Fisher information of the complete data space [2]. Sequential group updates yield a fast algorithm with tractable maximization steps. The algorithm was tested in the range of SNR from $-2 \mathrm{~dB}$ to $8 \mathrm{~dB}$. Wavelet decomposition on 6 scales was performed using the Daubechies compactly supported wavelet basis of filter length $N=4$. The step size of the algorithm (number of new wavelet coefficients in each cycle) was 10. The number of wavelet coefficients per bit was approximately 63 . In the GCA algorithm 4 bits were jointly updated ( 2 adjacent bits per user).

Figure 1 shows bit error probability results for the case of nearly equal power users: $E_{1}-E_{2}=0.7 \mathrm{~dB}$, where $E_{l}$ is the $l$-th user power in $d B$. The results indicate that both users practically achieve the single user BPSK bound. For comparison, the results of a conventional receiver for user 1 are shown. The conventional receiver was implemented with the single user algorithm of [1] assuming known gain and time delay. Clearly, the conventional receiver's performance degrades compared to the multiuser receiver with the increase of SNR, although it gives comparable performance at the lower end of the SNR range.

Figure 2 shows results of the same algorithm for the unequal power case. Here $E_{1}-E_{2}=3.7 \mathrm{~dB}$. Similar to the first case, both users achieve the single user bound. This shows that the near-far problem has been alleviated. Results of the conventional receiver for user 2 are shown for comparison. The performance degradation of the conventional receiver with respect to the multiuser receiver over the whole SNR range is clearly evident.

\section{REFERENCES}

[1] I. Sharfer, A.O. Hero, "A Maximum Likelihood CDMA Receiver Using the EM Algorithm and the Discrete Wavelet Transform", in Proc. ICASSP, (Atlanta, GA), May 1996.
[2] J.A. Fessler, A.O. Hero, "Space-alternating generalized expectation-maximization algorithm", IEEE Trans. ASSP, 42(10):2664-2677, Oct. 1994.

[3] S. Verdu, "Minimum probability of error for asynchronous Gaussian multiple-access channels", IEEE Trans. IT, 32(1):85-96, Jan. 1986.

[4] R. Lupas, S. Verdu, "Linear multiuser detectors for synchronous code-division multiple-access channel", IEEE Trans. IT, 35(1):123-136, Jan. 1989.

[5] I. Daubechies, "Orthonormal bases of compactly supported wavelets", Comm. Pure Appl. Math., 41(1):909$996,1988$.

[6] I. Sharfer, Recursive Algorithms for Digital Communica. tions Using the Discrete Wavelet Transform, $\mathrm{PhD}$ thesis, University of Michigan, 1996.

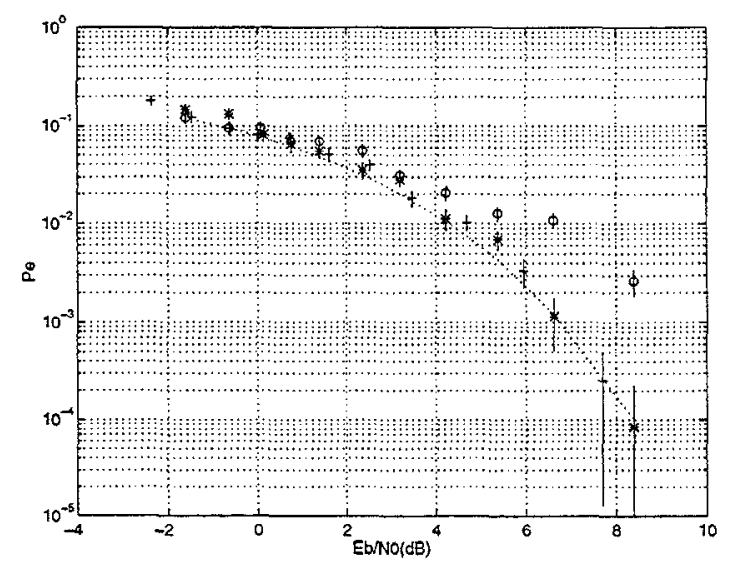

Figure 1. Bit error probability vs. SNR, 2 users, GCA algorithm $E_{1}-E_{2}=0.7 \mathrm{~dB},{ }^{*}$ ': user 1 ; $^{\prime} t^{\prime}$ : user 2; 'o': conventional receiver for user 1; dotted: BPSK error bound.

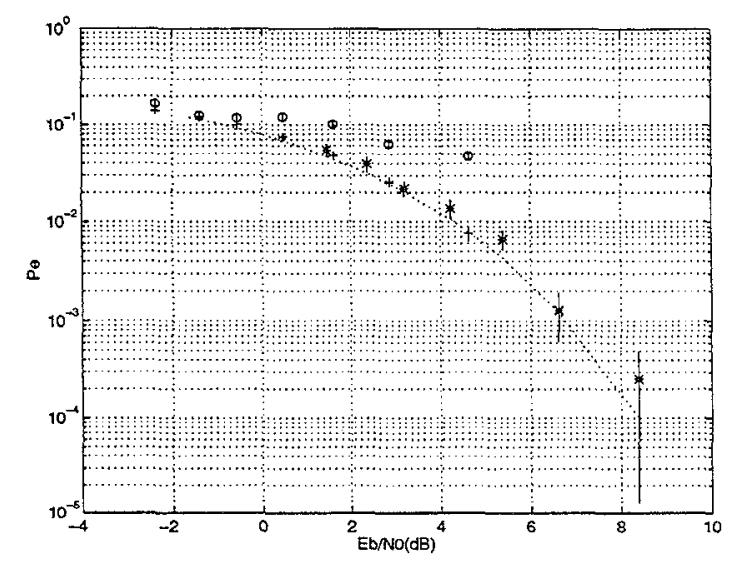

Figure 2. Bit error probability vs. SNR, 2 users, GCA algorithm $E_{1}-E_{2}=3.7 \mathrm{~dB}$, '*': user 1; '+': user 2; 'o': conventional receiver for user 2; dotted: BPSK error bound. 